\title{
BMJ Open Burden of musculoskeletal-related disorders resulting from non-fatal road traffic collisions in Africa: a protocol of a systematic review
}

\author{
Mohamed Yusuf (D), Tadesse Gebrye, Francis Fatoye
}

To cite: Yusuf M, Gebrye T, Fatoye $\mathrm{F}$. Burden of musculoskeletal-related disorders resulting from nonfatal road traffic collisions in Africa: a protocol of a systematic review. BMJ Open 2019;9:e032687. doi:10.1136/ bmjopen-2019-032687

- Prepublication history and additional material for this paper are available online. To view these files, please visit the journal online (http://dx.doi org/10.1136/bmjopen-2019032687).

Received 01 July 2019 Revised 05 October 2019 Accepted 07 October 2019
Check for updates

(C) Author(s) (or their employer(s)) 2019. Re-use permitted under CC BY-NC. No commercial re-use. See rights and permissions. Published by BMJ.

Manchester Metropolitan University, Manchester, UK

Correspondence to

Mohamed Yusuf;

m.yusuf@mmu.ac.uk

\section{ABSTRACT}

Introduction In addition to their high mortality rates, road traffic collisions (RTC) are considered a burden within those who do survive. In Africa, injuries related to RTC have been identified as the 10th most common cause of disability-adjusted life years lost for all ages and categories. Moreover, non-fatal RTC are the biggest cause associated with the burden of musculoskeletal disorders (MSD) in Africa. Despite this, the epidemiology of MSD related to non-fatal RTC within this region is still unknown. The objective of this review is to identify and summarise available data regarding the prevalence, incidence and patterns of MSD resulting from non-fatal RTC in Africa. Methods and analysis A systematic review of observational epidemiological studies will be conducted. On October 2019, MEDLINE, Embase, CINAHL Plus, PsycINF0, Web of Science, Global Health and African Journals OnLine will be searched for literature that is in English and published between 1990 and 2018. Two reviewers (MY ad TG) will independently screen results and extract the data, with a third reviewer (FF) resolving any discrepancies through discussion. Additionally, two reviewers (MY and TG) will independently assess the quality of the eligible studies using the US National Institutes of Health National Heart, Lung, and Blood Institute Quality Assessment Tool.

Within the eligible studies, data on citation, methods and results will be extracted. Extracted data will be analysed using a narrative synthesis. Findings will be presented in tables, with the main outcomes being prevalence, incidence and disease pattern of MSD and determinants of non-fatal RTC. The final systematic review will be conducted as per the Preferred Reporting Items for Systematic Reviews and Meta-Analyses guideline. Ethics and dissemination As individual patients will not be involved in this study, no ethical approval will be sought. Findings from this review will be disseminated through peer-reviewed journals and relevant conferences. Trial registration number CRD42019139538

\section{BACKGROUND}

Over the past few decades, due to the dedicated attention, resources and progress, the global burden of disease has shifted from communicable diseases to non-communicable diseases and injuries. ${ }^{1}$ Surpassing
Strengths and limitations of this study

This systematic review protocol follows the Preferred Reporting Items for Systematic Reviews and MetaAnalyses for Protocols guideline.

- This protocol prevents the potential for duplication and allows for transparency in the research process.

- This systematic review will be the first to synthesis the prevalence, incidence and patterns of musculoskeletal disorders from non-fatal road traffic collisions (RTC) in Africa.

- It is hoped that findings from this review will provide evidence for policymakers and stakeholders to put in place prevention strategies to mitigate the burden associated with RTC within this region.

- It is likely that there will be some under-reporting in the incidence of RTC within Africa.

tuberculosis and HIV/AIDS, road traffic collisions (RTC) are the eighth leading cause of death and the number one leading cause of death for children and young adults aged between 5 and 29 years. ${ }^{2}$ These rates are at their highest in Africa, where there are 26.6 deaths per 100000 people; this is substantially high in comparison to Europe where the death rate is at its lowest (9.3 deaths per 100000 people). Further, the WHO predicts that by 2030 injuries from RTC will be the seventh leading cause of deaths globally. ${ }^{3}$

In addition to their high mortality rates, RTC are considered a burden within those who do survive. They have a significant impact on quality of life, clinical and financial burden, and therefore are a major public health problem. ${ }^{4-6}$ In Africa, injuries related to RTC have been identified as the 10th most common cause of disability-adjusted life years lost for all ages and categories. ${ }^{1}$ Within Africa, non-fatal RTC are the biggest cause of burden associated with musculoskeletal disorders (MSD); this rate is more than the combined burden of the three big non-traumatic MSD (low back pain, osteoarthritis and 
rheumatoid arthritis combined). ${ }^{7}$ Despite such burden, the epidemiology of MSD related to non-fatal RTC within this region is still unknown.

There has been previous research evaluating the burden of RTC in Africa, ${ }^{89}$ though, there has not yet been a systematic review synthesising the epidemiology of MSD resulting from non-fatal RTC within Africa. Knowing the burden of these disorders is vital as it provides baseline data by evaluating what is already known, thus providing evidence for policymakers and stakeholders, including the WHO and the US Centers for Disease Control, ${ }^{10} 11$ who wish to undertake prevention strategies to mitigate the burden associated with RTC within Africa.

\section{Aims and objectives}

To establish the burden associated with MSD relating to non-fatal RTC, we aim to examine the burden of MSD-related RTC, with the objective of identifying and summarising available data regarding the prevalence, incidence and patterns of MSD resulting from non-fatal RTC in the different type of road users (pedestrian, passenger, driver and cyclists) within Africa.

\section{METHODS}

This systematic review protocol follows the Preferred Reporting Items for Systematic Reviews and Meta-Analyses (PRISMA) for Protocols statement. ${ }^{12}$ Additionally, the final systematic review will be conducted as per the PRISMA guideline. ${ }^{13}$

\section{Eligibility criteria}

Studies will be included if they were conducted in an African country between 1990 and 2018, as within this period data capture reporting of non-fatal RTC is anticipated to be of good quality. ${ }^{8}$ Studies that are of the following observational design will also be included: cross-sectional, prospective cohort, retrospective cohort and case control, and also if they used data from population-based or registry-based data systems. Additionally, studies should estimate the number of MSD due to non-fatal RTC in pedestrians, passengers, cyclists or drivers. Studies will be excluded if they referred to other non-road transportation systems, such as water and air, and if they were a review, opinion piece, commentary, case study and case series. If studies looked at other non-MSD, such as neurological injuries or wound infection, they will also not be included in the review.

In this review, our case definition is non-fatal injuries, including minor injuries with disabilities of short duration to severe injuries with lifelong disabilities, resulting from RTC by various road user types (pedestrians, motorised four-wheeler occupants, motorised two-wheeler and three-wheeler users, and cyclists). ${ }^{2}$ Further, we define MSD as soft-tissue injuries or pain within the skeletal systems that may be associated with injury affecting muscles, bones, ligaments, cartilages and so on. ${ }^{13}$

\section{Information sources and search strategy}

On October 2019, the following electronic databases will be searched for relevant literature: MEDLINE, Embase, CINAHL Plus, PsycINFO, Web of Science, Global Health and African Journals OnLine. With the help of the University librarian, a search strategy will be set in place; the key search concepts will be road traffic collision and injuries, Africa, observational data design and musculoskeletal injuries-see online supplementary file 1 for full search terms. Two reviewers (MY and TG) will initially execute the search strategy. Search results will be limited to the English language.

\section{Study selection}

All the abstracts found in the search results will be pooled and managed in Zotero referencing software, after that duplicates will be removed from this list. Two reviewers (MY and TG) will evaluate the titles and abstracts of the search results against the prespecified extraction criteria. Subsequently, both reviewers will independently screen the full articles against the eligibility criteria, any disagreements between the two reviewers will be resolved by the third reviewer (FF) through a discussion.

\section{Data collection process}

Based on the extraction list, two reviewers (MY and TG) will independently extract the relevant data into an Excel spreadsheet (V.2016, Microsoft). Both reviewers will independently cross-check extracted data, if consensus is not reached, then a third reviewer (FF) will resolve discrepancies between the two reviewers through discussion.

Once the eligible studies are finalised, the quality of studies will be appraised by two reviewers (MY and TG) using the US National Institutes of Health National Heart, Lung, and Blood Institute Quality Assessment Tool (NIH, 2014). This widely used assessment tool determines the quality of observational studies. In the form of questions, there are 12-14 items within the appraisal tool. These include sample size, population sampling, follow-up period, measurements and outcomes of interest. Independently, reviewer 1 and 2 (MY and TG) will subjectively determine the presence of these elements; potential answers for the questions include yes, no or other (cannot determine, not applicable or not reported). If there is no consensus between the two reviewers, then a third reviewer (FF) will resolve discrepancies through a discussion. The summary of each study will be calculated and expressed as a percentage. The interpretation for the scale will be categorised into four groups: poor $(0 \%-25 \%)$, fair $(25 \%-$ $50 \%)$, good $(50 \%-75 \%)$ or excellent $(75 \%-100 \%)$.

\section{Data items}

There will be four categories of extracted data, this includes data on citation, study, methods and results (see box 1 for extraction list and coding system). In the citation category, data on title, lead author, year of publication and journal will be extracted. For the study 


\section{Box 1 Extraction list and coding system}

\section{Citation}

a. Title of article

b. Lead author

c. Year published

d. Journal

2. Study

a. Aims/goals/objectives

3. Methods

a. Country of study

b. Study design

c. Study period

d. Sample size

e. Case definition

4. Results

a. Participation rate

b. Demographics of included cohort

c. Total number of non-fatal road traffic collisions (by subgroups)

d. Prevalence, incidence and disease patterns of musculoskeletal disorders

section, study aims or goals will be extracted. For the methods section, country of study, study design, study period, sample size and case definition will be extracted. Data extraction in the results section involves extracting data related to the reported RTC, this involves participation rate, demographics of the included cohort and total number of non-fatal RTC (per vehicle, per road users and per demographics). Additionally, data on prevalence, incidence and disease pattern of MSD will be extracted, and if available, data on the determinants of RTC will also be extracted. Data will be grouped by country of study, year, age, sex, type of MSD and type of RTC determinant. We define prevalence as the proportion of individuals in a population who have non-fatal RTC-related MSD in a specific time period, while incidence rate is the number of new cases who have non-fatal RTC-related MSD during a certain period of time. $^{14}$

\section{Analysis}

Extracted data will be analysed using a narrative synthesis. Findings will be presented in tables, with the main outcomes being prevalence, incidence and disease pattern of MSD. Further, data will be grouped by country of study, year, age, sex and type of MSD.

\section{Ethics and dissemination}

As individual patients will not be involved in this study, no ethical approval will be sought. Findings from this review will be disseminated through peer-reviewed journals and relevant conferences.

\section{Patient and public involvement}

There was no patient or public involvement in any phase of this study, this included the development of the research question, the analysis and the conclusions.

\section{DISCUSSION}

This systematic review will provide a synthesis of the current evidence pertaining to the prevalence and incidence of non-fatal RTC in Africa. Conclusion drawn from this is envisaged to inform future policies and public health prevention strategies to minimise the burden related to RTC in Africa. This review has some limitations. First, it is likely that there will be some under-reporting in the incidence of RTC within Africa. ${ }^{8}$ Second, it is likely that studies will be heterogeneous in quality and design.

Contributors FF is a guarantor of this protocol. All the authors have made substantive intellectual contributions to the development of this review. MY, TG and FF were involved in conceptualising the review. MY developed the protocol and was involved in the writing of the manuscript. All the authors contributed to the editing.

Funding The authors have not declared a specific grant for this research from any funding agency in the public, commercial or not-for-profit sectors.

Competing interests None declared.

Patient consent for publication Not required.

Provenance and peer review Not commissioned; externally peer reviewed.

Open access This is an open access article distributed in accordance with the Creative Commons Attribution Non Commercial (CC BY-NC 4.0) license, which permits others to distribute, remix, adapt, build upon this work non-commercially, and license their derivative works on different terms, provided the original work is properly cited, appropriate credit is given, any changes made indicated, and the use is non-commercial. See: http://creativecommons.org/licenses/by-nc/4.0/.

ORCID iD

Mohamed Yusuf http://orcid.org/0000-0002-9339-4613

\section{REFERENCES}

1 Murray CJL, Ezzati M, Flaxman AD, et al. GBD 2010: design, definitions, and metrics. The Lancet 2012;380:2063-6.

2 Wolrd Health Organisation. Global status report on road safety 2018, 2018. Available: https://www.who.int/violence_injury_prevention/ road_safety_status/2018/en/

3 Wolrd Health Organisation. Global status report on road safety 2018, 2018. Available: http://www.who.int/mediacentre/factsheets/fs358/ en/

4 Rissanen R, Berg H-Y, Hasselberg M. Quality of life following road traffic injury: a systematic literature review. Accid Anal Prev 2017;108:308-20.

5 Olukoga A, Harris G. Field data: distributions and costs of road-traffic fatalities in South Africa. Traffic Inj Prev 2006;7:400-2.

6 Dhondt S, Macharis C, Terryn N, et al. Health burden of road traffic accidents, an analysis of clinical data on disability and mortality exposure rates in Flanders and Brussels. Accid Anal Prev 2013;50:659-66.

7 Institute for Health Metrics and Evaluation (IHME). GBD compare data visualization 2018. Seattle, WA: IHME, University of Washington, 2018. http://vizhub.healthdata.org/gbd-compare

8 Adeloye D, Thompson JY, Akanbi MA, et al. The burden of road traffic crashes, injuries and deaths in Africa: a systematic review and meta-analysis. Bull World Health Organ 2016;94:510-21.

9 Vissoci JRN, Shogilev DJ, Krebs E, et al. Road traffic injury in subSaharan African countries: a systematic review and summary of observational studies. Traffic Inj Prev 2017;18:767-73.

10 Organization WH. Global status report on road safety 2018 . World Health organization, 2018.

11 Centre for Disease Control. Road traffic injuries and Deaths-A global problem, 2018. Available: https://www.cdc.gov/features/ globalroadsafety/index.html

12 Moher D, Shamseer L, Clarke M, et al. Preferred reporting items for systematic review and meta-analysis protocols (PRISMA-P) 2015 statement. Syst Rev 2015;4:1.

13 Broniecki M, Esterman A, May E, et al. Musculoskeletal disorder prevalence and risk factors in ambulance officers. J Back Musculoskelet Rehabil 2010;23:165-74.

14 Rothman KJ, Greenland S, Lash TL. Modern epidemiology. Lippincott Williams \& Wilkins, 2008. 